\title{
Design of Low-Carbon Supply Chain Under Emission Trading Scheme
}

\author{
lisi ke_ ${ }_{-}{ }^{1}$ hanping hou_ ${ }^{2}$ \\ Beijing jiaotong University Beijing,China \\ kelisi123@126.com
}

\begin{abstract}
In the context of climate change, greenhouse gas has aroused the general attention. In terms of supply chain management, managers tend to focus on the design of low -carbon supply chain. As the government enforced the emission trading scheme, foreign managers have considering the emission trading into the design of supply chain, while the development of emission trading is still exploring in china, and the initial allocation of the emission allowance is the first problem to solve, it's necessary to discuss the design of low-carbon supply chain considering different allocations of emission allowance under emission trading.
\end{abstract}

Key words: low-carbon supply chain; emission trading scheme; emission allowance.

\section{Introduction}

In context of climate change greenhouse gas emission has aroused the general attention. In terms of supply chain management, managers have begun to pay close attention to the design of lowcarbon supply chain. Many foreign scholars embedded carbon trading into the design of supply chain. As known, emission trading is a kind of market mechanism which allows the companies to buy emission allowance from those who pollute less in order to stay below their emissions limit or cap which usually imposed by a central authority .while the development of emission trading is still exploring in china, and the initial allocation of the emission allowance is the first problem to solve, In a result, according to the existing domestic and overseas research, we propose a mix-integer programming model to formulate low-carbon supply chain.

\section{Literature review}

The low-carbon supply chain has been widely studied in response to stricter environmental regulations in recent years. At present, they can generally be divided into four categories: management the green supply chain from the perspective of low-carbon , operation of low-carbon products supply chain, management of low-carbon supply chain, carbon management of supply chain, which present various strategies and principles about how to design low-carbon supply chain. However, few studies think about using the emission trading market as a tool to quantify the design of low-carbon supply chain. The trading of emissions under a "cap-and-trade" system places supply chains mangers in a different situation compared with the traditional control approach.

First, corporations must consider the available alternatives (options) that might allow them to meet the cap. Second, they must compare the cost of adopting some of these options with the current trading price of the emissions in question. 
While the design of low-carbon supply chain is almost equivalent to the design of green supply chain to some extent, and most of these studies already embedded carbon trading into the design of supply chain ,yet not comprehensive for merely considering the design of supply chain with distributing the allowance for free. In practice, the EU ETS rules specify that in the first trading period, only a small percentage of allowances may be auctioned, and at least $95 \%$ of allowances should be allocated for free. In the second period, the amount to be given away for free has to be at least 90\%.Moreover, Economist believe that auction is the most efficient way of emission allowance distribution, while it would be easier for the governor to promote the emission trading scheme if the allowance are free to get for the emitters.

In summary, we believe it's necessary to combine emission trading and the allocation of allowance into the design of low-carbon supply chain.

\section{Model formulation}

\section{1 model description}

The proposed mathematical model focuses on studying the impact of production, transportation and warehousing activities with a carbon emission constraint. The supply chain network consists of a set of plants of various production capacities, a set of distribution centers (DCs) of various throughput capacities, a set of retailers, and a set of product types. The problem is to decide which plants and DCs to open, how the DCs are assigned to the plants, and how the DCs distribute multiple types of products to satisfy retailers' demands, in such a way that the total facility opening and products distribution costs are minimized and total carbon emission is not more than a predetermined emission cap. Retailers' demands are assumed to be deterministic, and the plants and DCs have limited production and throughput capacities. Throughout this paper, we use the words warehouse and DC interchangeably, while we use the word facility to refer to a plant, warehouse, or retailer.

\subsection{Notation}

$I$ : set of retailers, indexed by $\mathrm{i}$

$J$ : set of warehouses, indexed by i

$K$ : set of plants, indexed by $\mathrm{k}$

$\tilde{J}$ : set of warehouses sizes, indexed by $\mathrm{j}$

$\tilde{K}:$ set of plants sizes, indexed by $\mathrm{k}$

$L:$ set of products, indexed by 1

$R$ : average expected cost of carbon credits, in $¥ /$ ton $\mathrm{co}_{2}$

\section{Parameter}

$f_{k}^{\tilde{k}}$ : fixed-cost to run a plant of size $\tilde{k}$ at location $k$

$g_{j}^{\tilde{j}}$ : fixed cost to open and operate a DC of size $\tilde{j}$ at location $j$

$a_{i l}$ : demand of retailer $i$ for product type $l$

$c_{l}$ : distribution cost of product type $l$ per unit distance

$w^{\tilde{j}}$ : size, in $\mathrm{ft} 2$, of DC $\tilde{j}$

$p^{\tilde{k}}$ : size, in $\mathrm{ft} 2$, of plant $\tilde{k}$

$q_{l}$ : area, in $\mathrm{ft} 2$, required to produce one unit of product type $l$ at any plant

$s_{l}$ : area, in $\mathrm{ft} 2$, required to store a unit of product type $l$ at any DC

$d_{j k}^{p}$ : distance, in miles, between a plant at location $k$ and $\mathrm{DC}$ at location $j$

$d_{i j}^{w}$ : distance, in miles, between retailer $i$ and a DC at location $j$ $e^{c a p}$ :maximum amount (in tons) of carbon dioxide $(\mathrm{CO} 2)$ that can be emitted (allocated by the government) 
$e^{c u r}$ : amount of carbon dioxide (CO2), in

tons, that is currently emitted

$\alpha_{p}$ :CO2 emissions factor of a facility, in ton per $\mathrm{kWh}$ of operation

$\alpha_{w}: \mathrm{CO} 2$ emissions factor of a warehouse, in tons per $\mathrm{ft} 3$

$\alpha_{t}$ : CO2emissions factor for transportation, in tons per mile

$M$ : very large number

\section{Decision Variables}

$X_{i j l}$ : total number of units of product type distributed

to retailer from warehouse

$Y_{j k l}$ : total number of units of product type shipped from plant to warehouse

$U_{j}^{\tilde{j}}=\left\{\begin{array}{l}1, \text { if a DC of size } \tilde{j} \text { is opened at location } j \\ 0, \text { otherwise }\end{array}\right.$

$V_{k}^{\tilde{k}}=\left\{\begin{array}{l}1, \text { if a plant with production capacity } \tilde{k} \text { at location } k \\ 0, \text { otherwise }\end{array}\right.$

$\tilde{X}_{i j}=\left\{\begin{array}{l}1, \text { if } \mathrm{CD} i \text { distributes product to retailer } j \\ 0, \text { otherwise }\end{array}\right.$

$\tilde{Y}_{j k}=\left\{\begin{array}{l}1, \text { if plant } k \text { distribute products to } \mathrm{CD} j \\ 0, \text { otherwise }\end{array}\right.$

\subsection{The Model}

$$
\begin{aligned}
& \min \left\{\sum_{j \in J} \sum_{j \in \tilde{J}} g_{j}^{\tilde{j}} U_{j}^{\tilde{j}}+\sum_{j \in \tilde{j}} \sum_{i \in l} \sum_{j \in J} \sum_{l \in L} c_{l} d_{i j}^{w} X_{i j l}+\sum_{k \in K} \sum_{j \in J} \sum_{l \in L} c_{l} d_{j j k}^{p} Y_{j k l}+\right. \\
&\left.\sum_{k \in K} \sum_{\hat{k} \in \tilde{K}} f_{k}^{\tilde{k}} V_{k}^{\tilde{k}}+R\left(e^{c u r}-e^{c a p}\right)\right\} \\
& \text { s.t. } \quad \sum_{j \in J} X_{i j l}=a_{i l}, \quad \forall i \in I, l \in L \\
& \sum_{i \in I} \sum_{l \in L} s_{l} X_{i j l} \leq w^{\tilde{j}} U_{j}^{\tilde{j}}, \quad \forall j \in J, \tilde{j} \in \tilde{J} \\
& \sum_{i \in I} X_{i j l} \leq \sum_{k \in K} Y_{j k l}, \quad \forall j \in J, l \in L \\
& \sum_{j \in J} \sum_{l \in L} q_{l} Y_{j k l} \leq p^{\tilde{k}} V_{k}^{\tilde{k}}, \quad \forall k \in K, \tilde{k} \in \tilde{K}
\end{aligned}
$$

$$
\begin{aligned}
& \alpha_{p} \sum_{k \in K} \sum_{\tilde{k} \in \tilde{K}} p^{\tilde{k}} V_{k}^{\tilde{k}}+\alpha_{w} \sum_{j \in J} \sum_{j \in J} w^{\tilde{j}} U_{j}^{\tilde{j}}+ \\
& \alpha_{t} \sum_{i \in l} \sum_{j \in J} \sum_{k \in K}\left(d_{j k}^{p} \tilde{Y}_{j k}+d_{i j}^{w} \tilde{X}_{i j}\right)=e^{c u r} \\
& X_{i j l} \leq M \tilde{X}_{i j}, \quad \forall i \in I, j \in J, l \in L \\
& Y_{j k l} \leq M \tilde{Y}_{j k}, \quad \forall i \in I, j \in J, l \in L \\
& X_{i j l} \geq 0, \quad \forall i \in I, j \in J, l \in L \\
& Y_{j k l} \geq 0, \quad \forall i \in I, j \in J, l \in L \\
& \tilde{X}_{i j}=\{1,0\}, \quad \forall i \in I, j \in J \\
& \tilde{Y}_{j k}=\{1,0\}, \quad \forall i \in I, j \in J \\
& U_{j}^{\tilde{j}}=\{1,0\}, \quad \forall j \in J, \tilde{j} \in \tilde{J} \\
& V_{k}^{\tilde{k}}=\{1,0\}, \quad \forall k \in K, \tilde{k} \in \tilde{K}
\end{aligned}
$$

This model is formulated under the assumption that emission allowance is allocated for free. The objective function equation (1) minimizes the sum of the fixed cost, distribution costs and allowance cost traded on market. Constraint set equation (2) ensures that the demand of each retailer is satisfied by the open DCs. Constraint set equation (3) ensures that the demands of retailers that are supplied by open DCs do not exceed the throughput capacity of any of these DCs. Constraint set equation (4) ensures that total flow of product $l$ that enters $\mathrm{DC}_{j}$ from all plants does not exceed the flow that leaves the DC to all retailers. Constraint set equation (5) represents the capacity restriction of plant of size in terms of the amount of product it can handle. Constraint set equation (6) ensures that the total carbon dioxide emission does not exceed an emission cap, which is usually determined by environmental regulations. The two new variables (and) that are used in constraint set equations (6)-(8) are auxiliary binary variables that take value one with positive flow between facilities, which is guaranteed by equations (7)-(8), where $M$ refers to satisfactorily large number. Constraint set equations (9)-(10) and (11)-(14) enforce the non-negativity 
and binary restrictions on the decision variables.

$$
\begin{aligned}
& \min \left\{\begin{array}{c}
\sum_{j \in J} \sum_{j \in J} g_{j}^{\tilde{j}} U_{j}^{\tilde{j}}+\sum_{i \in l} \sum_{j \in J} \sum_{l \in L} c_{l} d_{i j}^{w} X_{i j l}+\sum_{k \in K} \sum_{j \in J} \sum_{l \in L} c_{l} d_{j k}^{p} Y_{j k l}+ \\
\sum_{k \in K} \sum_{k \in \tilde{K}} f_{k}^{\tilde{k}} V_{k}^{\tilde{k}}+R^{a}\left(e^{c a p}\right)+R\left(e^{c u r}-e^{c a p}\right)
\end{array}\right\}(1) \\
& \text { s.t. } \quad(2)-(14) \\
& \sum_{k \in K} \sum_{\tilde{k} \in \tilde{K}} f_{k}^{\tilde{k}} V_{k}^{\tilde{k}}=H_{K} \\
& \sum_{j \in J} \sum_{\tilde{j} \in \tilde{J}} g_{j}^{\tilde{j}} U_{j}^{\tilde{j}}=H_{J} \\
& \sum_{i \in I} \sum_{j \in J} \sum_{l \in L} c_{l} d_{i j}^{w} X_{i j l}+\sum_{k \in K} \sum_{j \in J} \sum_{l \in L} c_{l} d_{j k}^{p} Y_{j k l}=H_{T}
\end{aligned}
$$

Here we consider an allowance auction whose objective is to maximize the revenue plus the combined amounts bid by the supply chain. The new objective function equation is familiar with the original equation except the part which represents the cost of emission allowance by auction. $R^{a}$ is the price of unit ton $\operatorname{co} 2$ that the emitters would like to bid for the emission allowance on the carbon market. The price prediction of allowance in different markets is an important issue but beyond the scope of this paper.

We assume that the price $R^{a}$ is relevant with the cost and the amount of emissions. In this paper, we briefly suppose that the price is a one-order function $R^{a}=f\left(e^{c u r}, H_{K}+H_{J}+H_{T}\right)$ and $e^{c u r}$ represents the total amount of the supply chain emissions and has a significant impact on the bid price of the emission allowance, excess emission levels would impose the managers to pay more for the allowance. $H_{K}+H_{J}+H_{T}$ represents the total cost of formulating the supply chain ,and it would harm the supply chain' ability to bid, if the total cost is too high. We believe that the design of low-carbon supply chain will gain in richness and mind share if leveraging the opportunities offered by carbon trading markets for those companies pursuing a green strategy or having to regulate their GHGs emissions.

\section{Conclusion}

With the proposed model, we come up with a new model under the condition that the emission allowance is assigned by auction. It can help the managers to make a rational tradeoff between cost saving and emission reduction under the two different markets environment. Policies defining the allowance allocation can affect the intended goals such as controlling emissions, reducing total cost at a reasonable level of a low-carbon supply chain.

\section{ACKNOWLEDGEMENT}

This study was supported by the State Key Program of National Natural Science of China (Grant NO.71132008), and partially funded by the Humanities and Social Science Project of Education Ministry (10YJA630059) in China.

\section{Reference}

[1] Zhang Xin, "Preliminary Discussion on Low Carbon Supply Chain," Storage Transportation \& Preservation of Commodities, PP. 80-82,2011.

[2] Ali Diabat, David Simchi Levi, "A Carbon-Capped Supply Chain Network Problem," IEEE, IEEM, pp.524-527, 2009.

[3] Samir Elhedhi, Ryan Merrik, "Green Supply Chain Network Design to Reduce Carbon Emissions," Transportation Research part D, pp.370379,2012 . 